\title{
PROPAGACIÓN VEGETATIVA DE Pinus patula SCHIEDE ex SCHLTDL. et CHAM. EN DIFERENTES SUSTRATOS
}

\section{VEGETATIVE PROPAGATION OF Pinus patula SCHIEDE ex SCHLTDL. et CHAM. IN DIFFERENT SUBSTRATES}

\section{Nohemí Escamilla-Hernández, Arnulfo Aldrete*, J. Jesús Vargas-Hernández, Ángel Villegas-Monter y Miguel Á. López-López}

Colegio de Postgraduados, Postgrado en Ciencias Forestales, Montecillo, Texcoco, Estado de México, México.

*Autor de correspondencia (aaldrete@colpos.mx)

\section{RESUMEN}

Pinus patula Schiede ex Schldl. et Cham. es una especie endémica de México utilizada en plantaciones forestales comerciales en Sudamérica y Sudáfrica. La propagación masiva de materiales mejorados de esta especie, a través de estacas enraizadas, permite aumentar su productividad y homogeneizar sus productos. Se compararon cinco sustratos a base de aserrín fresco y corteza de pino en diferentes proporciones (1:9, 3:7, 5:5, 7:3 y 9:1 v:v) para evaluar el enraizamiento de estacas de esta especie forestal. Se utilizó un diseño experimental de bloques completos al azar con cuatro repeticiones; la unidad experimental estuvo conformada por 25 estacas. Se analizaron las principales características físicas y químicas de los sustratos tales como porosidad total, porosidad de aireación, retención de humedad, densidad aparente, $\mathrm{pH}$, conductividad eléctrica y capacidad de intercambio catiónico al inicio y al final del experimento. Las variables evaluadas fueron la supervivencia, enraizamiento, presencia de callo, número y longitud de raíces primarias y presencia de raíces secundarias a las 20 semanas de establecido el experimento. El sustrato con la mayor proporción de aserrín (9:1) presentó el porcentaje de enraizamiento de las estacas más elevado (77 $\%$ ), en comparación con el testigo (1:9), donde se obtuvo $42 \%$. Se observó alta correlación $(0.97,0.97,0.90)$ del enraizado con el porcentaje de aserrín, densidad aparente y $\mathrm{pH}$, respectivamente. La mezcla de aserrín y corteza de pino (9:1) presentó características físicas y químicas apropiadas para la formulación de sustratos útiles para propagar $P$. patula mediante el enraizado de estacas.

Palabras clave: Pinus patula, aserrín de pino, corteza de pino, enraizado de estacas, propagación, viveros.

\section{SUMMARY}

Pinus patula Schiede ex Schldl. et Cham. is an endemic species of Mexico used in commercial forest plantations in South America and South Africa. Massive propagation of improved materials of this species, through rooted cuttings, allows the increase of productivity and homogenized products. Five substrates based on fresh sawdust and pine bark at different proportions $(1: 9,3: 7,5: 5,7: 3$ and 9:1 v:v) were compared to assess rooting of this forest species. A randomized complete block experimental design with four replications was used; the experimental unit was made up of 25 rooted cuttings. The main physical and chemical characteristics of the substrates such as total porosity, aeration porosity, moisture retention, bulk density, $\mathrm{pH}$, electrical conductivity and cation exchange capacity were analyzed at the beginning and the end of the experiment. The evaluated variables were survival, rooting, callus presence, number and length of primary roots and presence of secondary roots 20 weeks after the experiment was established. The substrate with the highest proportion of sawdust (9:1) had the highest percentage of rooting (77\%), compared to the control (1:9), where $42 \%$ were rooted. High correlation $(0.97,0.97,0.90)$ of rooting was observed with sawdust percentage, bulk density and $\mathrm{pH}$, respectively. The mixture of sawdust and pine bark (9:1) presented appropriate physical and chemical characteristics for the formulation of substrates useful for propagating Pinus patula by rooted cuttings.

Index words: Pinus patula, nurseries, pine bark, pine sawdust, propagation, rooted cuttings.

\section{INTRODUCCIÓN}

Pinus patula Schiede ex Schldl. et Cham. es una especie endémica de México (Farjon et al., 1997), importante tanto desde el punto de vista ecológico como comercial; es utilizada en los programas de reforestación debido a su gran abundancia, productividad de madera, captura de $\mathrm{CO}_{2}$ (Romo et al., 2014) y calidad de la madera (EscobarSandoval et al., 2018); presenta características ideales en la industria maderera como fuste recto, capacidad de poda natural y calidad de troza (Velázquez et al., 2004).

En los últimos 20 años se han iniciado programas de mejoramiento genético en México, a través de la selección fenotípica de árboles superiores y el establecimiento de ensayos de progenie (Salaya-Domínguez et al., 2012). Una vez seleccionados los mejores individuos, la idea es propagarlos de manera masiva (Méndez-Neri et al., 2020); una de las técnicas más utilizadas a nivel mundial para este propósito es el enraizado de estacas, que permite capturar y transferir a los rametos el potencial genético del árbol original (Zobel y Talbert, 1984).

En México se realizan esfuerzos para generar protocolos para el enraizamiento de estacas de Pinus patula; los mayores retos que se han presentado incluyen la definición del tamaño de estaca, estado de madurez de la 
planta madre, tipo de sustrato, crecimiento plagiotrópico y condiciones microambientales (Aparicio-Rentería et al., 2014).

El sustrato y el espacio del contenedor son factores fundamentales para la formación y desarrollo saludable de la raíz; debido a ello, los efectos combinados del entorno, como la cantidad de agua y frecuencia de aplicación del riego, deben ser bien comprendidos al seleccionar el sustrato a utilizar. Fonteno y Bilderback (1993) mencionaron que un sustrato debe ser el medio para: 1) poner disponible el agua 2) suministrar nutrimentos, 3) permitir el intercambio de gases entre la zona radicular y el exterior del sustrato y 4) dar soporte a la planta. De todas las propiedades del sustrato, las características físicas son las más importantes debido a que, una vez establecida la especie, difícilmente pueden manipularse (Abad et al., 2004) y deben permanecer estables durante su ciclo de producción (Wallach, 2008).

En el caso particular de las coníferas, el enraizamiento de estacas requiere, en su etapa inicial, condiciones favorables de humedad, sin llegar a la saturación o anegamiento del sustrato (Regonezi et al., 2010). La turba, vermiculita, perlita y arena son materiales comúnmente utilizados en la propagación vegetativa, ya sea de manera individual 0 en combinación en varias proporciones (Bielenin, 2003; Hamann et al., 1998; Rasmussen et al., 2009); sin embargo, el costo elevado de estos materiales los hace poco rentables (Aguilera-Rodríguez et al., 2016; Fain et al., 2008).

Por lo anterior, han surgido alternativas de sustratos locales que pueden usarse para el enraizamiento de estacas, como corteza de pino, cascarilla de arroz carbonizada y pino triturado (hojas, corteza y ramas). Con el uso de estos materiales se han reportado enraizamientos de 90 a 97 $\%$ en especies de la familia Cupressaceae (Stumpf et al., 1999; Witcher et al., 2014) y de 80 a $95 \%$ en el género Pinus (Alcantara et al., 2007; Browne et al., 2000; Henrique et al., 2006). Por su parte, Witcher et al. (2014) no encontraron diferencias al comparar cuatro sustratos compuestos por corteza, corteza/turba (1:1), pino triturado y turba/pino triturado (1:1) en el enraizamiento de Leyland cypress A.B. Jacks. \& Dallim. (Cupresaceae). En todos los tratamientos, el enraizamiento fue mayor de $90 \%$.

El aserrín de pino (Pinus sp.) es un material que tiene potencial como componente en la formulación de sustratos; es barato, se encuentra disponible localmente, es uniforme, es liviano y está libre de plagas (FregosoMadueño et al., 2017; Maher et al., 2008). Las propiedades físicas del aserrín dependen del tamaño de sus partículas, por lo que se recomienda que un 20 a $40 \%$ sean inferiores a $0.8 \mathrm{~mm}$ de longitud, con una densidad de 0.1 a $0.45 \mathrm{~g}$ $\mathrm{cm}^{-3}$. La porosidad total es superior a $80 \%$, la porosidad de retención de agua es de baja a media, pero su capacidad de aireación suele ser adecuada (Maher et al., 2008). Tchoundjeu et al. (2004) reportaron $79 \%$ de enraizamiento de Pausinystalia johimbe (K.Schum.) Pierre ex Beille (Rubiaceae), árbol africano, en este tipo de sustrato. Si bien la corteza de pino es un medio común para la propagación vegetativa, se requiere investigar sobre otros materiales disponibles localmente que puedan utilizarse para este propósito. El presente trabajo tuvo por objetivo comparar diferentes proporciones de aserrín y corteza de pino en la formulación de sustratos para lograr altas proporciones de enraizamiento de estacas de P. patula.

\section{MATERIALES Y MÉTODOS}

\section{Localización del estudio y condiciones de propagación}

La investigación se desarrolló en condiciones de invernadero en el Colegio de Postgraduados, Texcoco, Estado de México (190 27' 38.25" N y 98 54' 23.91" O, a $2240 \mathrm{~m}$ de altitud). Para la producción de setos, como fuente de estacas, se utilizó un lote de semillas proveniente del huerto semillero clonal Reserva Forestal Multifuncional de Aquixtla, Puebla, de primera generación, que incluyó los 11 mejores clones con base en los resultados de un ensayo de progenies (Salaya-Domínguez et al., 2012). La siembra se realizó en junio de 2017, en contenedores (tubetes) de $220 \mathrm{~cm}^{3}$. Las plantas crecieron en condiciones de invernadero hasta los siete meses, la altura promedio fue de $18 \mathrm{~cm}$; a esta edad se llevó a cabo la primera poda del tallo principal, en la que se eliminó la parte apical $(3 \mathrm{~cm})$ para promover la generación de nuevos brotes.

A los 15 días después de la poda las plantas se trasplantaron en macetas de $4 \mathrm{~L}$, que contenían como sustrato una mezcla de corteza de pino compostada, vermiculita, turba y perlita (60:15:15:10, v:V:V:v) con $7 \mathrm{~g} \mathrm{~L}^{-1}$ de fertilizante de liberación controlada Osmocote® (159-12 de N-P-K) de ocho a nueve meses de liberación, y se mantuvieron en condiciones de malla-sombra de $50 \%$. A los tres meses después de la poda se cosecharon los brotes nuevos con longitud de 7 a $9 \mathrm{~cm}$ y diámetro basal de 2.5 a $3 \mathrm{~mm}$ para establecer el experimento.

A los brotes se les eliminaron las acículas en los primeros $3 \mathrm{~cm}$ desde la base y se desinfectaron con Captan (N-triclorometiltio-4-ciclohexeno-1,2-dicarboximida) en dosis de $1.5 \mathrm{~g} \mathrm{~L}^{-1}$ durante $15 \mathrm{~min}$. Las estacas se trasplantaron en tubetes colocados en rejillas con 25 cavidades individuales de $220 \mathrm{~cm}^{3}$, a $3 \mathrm{~cm}$ de profundidad en el sustrato y se asperjaron con solución de fungicida (tiabendazol $1.5 \mathrm{~g} \mathrm{~L}^{-1}$ ) e insecticida (Imidacloprid $1.5 \mathrm{~g} \mathrm{~L}^{-1}$ ). 
Para mantener la humedad relativa cercana a saturación cada rejilla se colocó dentro de una bolsa de plástico de 90 $\mathrm{cm}$ de ancho por $120 \mathrm{~cm}$ de alto.

\section{Tratamientos y diseño experimental}

Se evaluaron cinco mezclas de sustratos con dos componentes (aserrín fresco de pino y corteza compostada de pino) en diferentes proporciones. Las proporciones de aserrín y corteza fueron: 1:9, 3:7, 5:5, 7:3 y 9:1. La corteza se cernió con una criba de $0.5 \mathrm{~cm}$. Los sustratos se desinfectaron con el fungicida Tecto [2-(4-Tiazolil)- $1 \mathrm{H}$ benzimidazol] en dosis de $1.5 \mathrm{~g} \mathrm{~L}^{-1}$ de agua; para ello, se llenaron los tubetes con el sustrato correspondiente a cada tratamiento, se regó a saturación, se dejó escurrir el exceso de agua y se aplicó el fungicida mediante una mochila aspersora. Las estacas se mantuvieron a una temperatura promedio entre el día y la noche de 20.8 y $22.5^{\circ} \mathrm{C}$, con humedad relativa mayor a $90 \%$. El diseño experimental utilizado fue de bloques completos al azar con cuatro repeticiones y la unidad experimental estuvo integrada por 25 estacas.

\section{Caracterización física y química de los sustratos}

Los análisis se llevaron a cabo al inicio y al final del experimento en el laboratorio de Física de Suelos del Colegio de Postgraduados, Campus Montecillo, mediante tres repeticiones (muestras) de cada sustrato. Las propiedades físicas analizadas fueron porosidad total (PT), porosidad de aireación (PA), porosidad de retención de humedad (PRH) y densidad aparente; las propiedades químicas fueron $\mathrm{pH}$, conductividad eléctrica (CE) y capacidad de intercambio catiónico (CIC).

Las características físicas de PT (79 a $91 \%$ se encuentran por arriba del intervalo para producción de planta en tubetes, mientras que la PA (16 a $24 \%$ ) se encuentra dentro de los límites recomendados (Mathers et al., 2007). Además, la PRH presentó valores más altos (55 a $74 \%$ ) y la DA en los sustratos $\mathrm{S}_{5}$ al inicio y $\mathrm{S}_{3}, \mathrm{~S}_{4}$ y $\mathrm{S}_{5}$ al final valores más bajos (0.14 a $0.18 \mathrm{~g} \mathrm{~cm}^{-3}$ ) del valor referido por Mathers et al. (2007) (Cuadro 1). Con respecto a las propiedades químicas, la CE presentó valores aceptables (0.1 a $0.4 \mathrm{dS} \mathrm{m}^{-1}$ ), pero el pH fue más ácido (3.8 a 4.6) y la CIC más elevada (20 a $31.7 \mathrm{cmol} \mathrm{kg}^{-1}$ ) que los valores sugeridos por Mathers et al. (2007) (Cuadro 2).

\section{Variables evaluadas}

A las 20 semanas de establecido el experimento se extrajeron las estacas del sustrato y se evaluaron las características de supervivencia, enraizamiento, presencia de callo, número y longitud de raíces primarias y presencia de raíces secundarias. Se consideraron estacas vivas aquellas que no presentaron tejido necrosado en ninguna parte. Las estacas con al menos una raíz de $1 \mathrm{~mm}$ de longitud se consideraron enraizadas. La presencia de callo se identificó como el abultamiento en la base del tallo, resultado de la división celular (Hartmann et al., 2014; Rasmussen et al., 2009); las raíces primarias son las que se originaron de la base o parte lateral del tallo y las secundarias las que se formaron a partir de las raíces primarias, las cuales presentaron longitud mínima de 0.5 a $1 \mathrm{~cm}$. Las raíces primarias se midieron con regla con aproximación a $1 \mathrm{~mm}$. A partir de las características evaluadas en cada estaca se obtuvo para cada unidad experimental el porcentaje de estacas vivas, con callo y enraizadas. Considerando únicamente a las estacas enraizadas, se obtuvo el número y longitud promedio de raíces primarias y el porcentaje de estacas con presencia de raíces secundarias.

\section{Análisis estadístico}

Se realizó análisis de varianza y comparación de medias de Tukey $(P \leq 0.05)$ mediante el procedimiento Mixed del programa SAS versión 9.3 (SAS Institute, 2011). El modelo utilizado en el análisis de varianza fue el siguiente:

$$
Y_{i j}=\mu+B_{i}+T_{j}+\varepsilon_{i j}
$$

Donde: $Y_{i j}$ es el valor observado de la variable en la i-ésima unidad experimental del $j$-ésimo tratamiento, $\mu$ es la media general, $B_{i}$ es el efecto del $i$-ésimo bloque, $T_{i}$ es el efecto del j-ésimo tratamiento y $\varepsilon_{i j}$ es el error experimental. Todas las variables respuesta, excepto número y longitud de raíz, se transformaron con la función arco seno antes del análisis de varianza y posteriormente los valores promedio fueron re-transformados a su valor original.

Se correlacionaron las propiedades físicas y químicas del sustrato con las variables respuesta de las estacas mediante los coeficientes de correlación de Pearson.

\section{RESULTADOS Y DISCUSIÓN}

De acuerdo con los análisis de varianza, no se presentaron diferencias en la supervivencia $(P=0.2890)$, número de raíces primarias $(P=0.3929)$, longitud de raíz $(0.1107)$ y presencia de raíces secundarias $(P=0.6528)$; sin embargo, sí hubo diferencias significativas en enraizamiento $(P=$ 0.0031) y presencia de callo $(P=0.0005)$ (Figura 1).

La supervivencia de las estacas fue mayor de 90 $\%$ en todos los sustratos (Figura 1A). Con respecto al enraizamiento, existió una correlación elevada (0.97) y directamente proporcional con el contenido de aserrín en 
Cuadro 1. Propiedades físicas de los sustratos utilizados para el enraizamiento de Pinus patula Schiede ex Schldl. et Cham.

\begin{tabular}{|c|c|c|c|c|c|c|c|c|}
\hline \multirow{2}{*}{$\begin{array}{l}\text { Sustratos } \\
\text { (aserrín:corteza) }\end{array}$} & \multicolumn{2}{|c|}{ PT } & \multicolumn{2}{|c|}{ PA (\%) } & \multicolumn{2}{|c|}{$\mathrm{PRH}$} & \multicolumn{2}{|c|}{$\mathrm{DA}\left(\mathrm{g} \mathrm{cm}^{-3}\right)$} \\
\hline & Inicial & Final & Inicial & Final & Inicial & Final & Inicial & Final \\
\hline$S_{1}(1: 9)$ & 79 & 85 & 21 & 18 & 58 & 68 & 0.24 & 0.22 \\
\hline$S_{2}(3: 7)$ & 82 & 85 & 24 & 19 & 58 & 67 & 0.22 & 0.20 \\
\hline$S_{3}(5: 5)$ & 79 & 91 & 24 & 20 & 55 & 71 & 0.20 & 0.18 \\
\hline $\mathrm{S}_{4}(7: 3)$ & 83 & 89 & 19 & 18 & 64 & 71 & 0.19 & 0.16 \\
\hline$S_{5}(9: 1)$ & 86 & 90 & 16 & 17 & 70 & 74 & 0.17 & 0.14 \\
\hline Referencia $^{+}$ & \multicolumn{2}{|c|}{$60-80$} & \multicolumn{2}{|c|}{$15-35$} & \multicolumn{2}{|c|}{$25-55$} & \multicolumn{2}{|c|}{ 0.19-0.70 } \\
\hline
\end{tabular}

PT: porosidad total, PA: porosidad de aireación, PRH: porosidad de retención de humedad, DA: densidad aparente. `Valor de referencia (Mathers et al., 2007).

Cuadro 2. Propiedades químicas de los sustratos utilizados para el enraizamiento de Pinus patula Schiede ex Schldl. et Cham.

\begin{tabular}{|c|c|c|c|c|c|c|}
\hline \multirow{2}{*}{$\begin{array}{l}\text { Sustratos } \\
\text { (aserrín:corteza) }\end{array}$} & \multicolumn{2}{|c|}{$\mathrm{pH}$} & \multicolumn{2}{|c|}{ CE $\left(d S m^{-1}\right)$} & \multicolumn{2}{|c|}{$\mathrm{ClC}\left(\mathrm{cmol} \mathrm{kg}^{-1}\right)$} \\
\hline & Inicial & Final & Inicial & Final & Inicial & Final \\
\hline$S_{1}(1: 9)$ & 3.8 & 4.1 & 0.4 & 0.3 & 35 & 27.8 \\
\hline$S_{2}(3: 7)$ & 3.8 & 4.5 & 0.3 & 0.2 & 29 & 31.7 \\
\hline$S_{3}(5: 5)$ & 3.8 & 4.2 & 0.2 & 0.2 & 29 & 30.1 \\
\hline $\mathrm{S}_{4}(7: 3)$ & 4.2 & 4.2 & 0.2 & 0.1 & 27 & 25.9 \\
\hline $\mathrm{S}_{5}(9: 1)$ & 4.4 & 4.6 & 0.3 & 0.1 & 19 & 20.0 \\
\hline Referencia $^{+}$ & \multicolumn{2}{|c|}{$5-6$} & \multicolumn{2}{|c|}{$<1.0$} & \multicolumn{2}{|c|}{$6-15$} \\
\hline
\end{tabular}

CE: conductividad eléctrica, CIC: capacidad de intercambio catiónico. `Valor de referencia (Mathers et al., 2007).

el sustrato (Cuadro 3). Al aumentar la proporción de aserrín en el sustrato de 10 a 90 \% el enraizamiento aumentó de 42 a $77 \%$ (Figura 1B). Este comportamiento también lo reportaron Tchoundjeu et al. (2004), quienes observaron incremento en el porcentaje de enraizamiento de Pausinystalia johimbe de 65 a 79 \% conforme incrementó el porcentaje de aserrín.

El enraizamiento observado en el presente estudio se encuentra por debajo de lo reportado por Browne et al. (2000) y Henrique et al. (2006) para Pinus banksiana Lamb. (87\%) y P. caribaea Morelet (95\%), en sustratos orgánicos de turba de musgo y cascarilla de arroz carbonizada; sin embargo, éste se podría incrementar al adicionar hormonas de enraizamiento, como ácido naftalenacético e indolbutírico, según lo reportan dichos autores.

La presencia de callo obedece a condiciones de aire en el sustrato durante la primera etapa de formación de raíces (King et al., 2011). Se observa correlación elevada (0.92) de la porosidad de aireación con la formación de callo (Cuadro 3); lo anterior puede explicarse por el hecho de que la corteza presenta partículas con tamaños superiores al aserrín, lo que contribuye a incrementar el espacio poroso y, por consecuencia, el desarrollo de callo (King et al., 2011; Mathers et al., 2007) (Figura 1C).

Con respecto al número y longitud de raíces primarias y presencia de raíces secundarias, Goldfarb et al. (1998) mencionaron que, adicional al enraizamiento de las estacas, la calidad del sistema radical juega un papel importante para la supervivencia en campo. Diferentes autores reportan, para especies de pino, de 1.4 a 2.0 raíces primarias (Rowe et al., 2002), $3.3 \mathrm{~cm}$ de longitud de raíz (Majada et al., 2011) y de 19.4 a $25.8 \%$ de plantas con raíces secundarias (Rivera-Rodríguez et al., 2016) al enraizar estacas en sustratos convencionales (perlita, vermiculita y turba). En el presente estudio se encontraron valores promedio iguales o mayores que los reportados anteriormente, con 1.7 a 2.1 raíces primarias por estaca, longitud de 5.3 a $6.7 \mathrm{~cm}$ y más de $60 \%$ de las estacas enraizadas presentaron raíces secundarias (Cuadro 4).

Las características físicas del sustrato influyen en el proceso de enraizamiento (Ragonezi et al., 2010). Se observó correlación positiva entre el enraizamiento (de 42 a $77 \%$ ) y la porosidad de retención de humedad (de 58 a 70 \%) y de forma negativa con la porosidad de aireación 

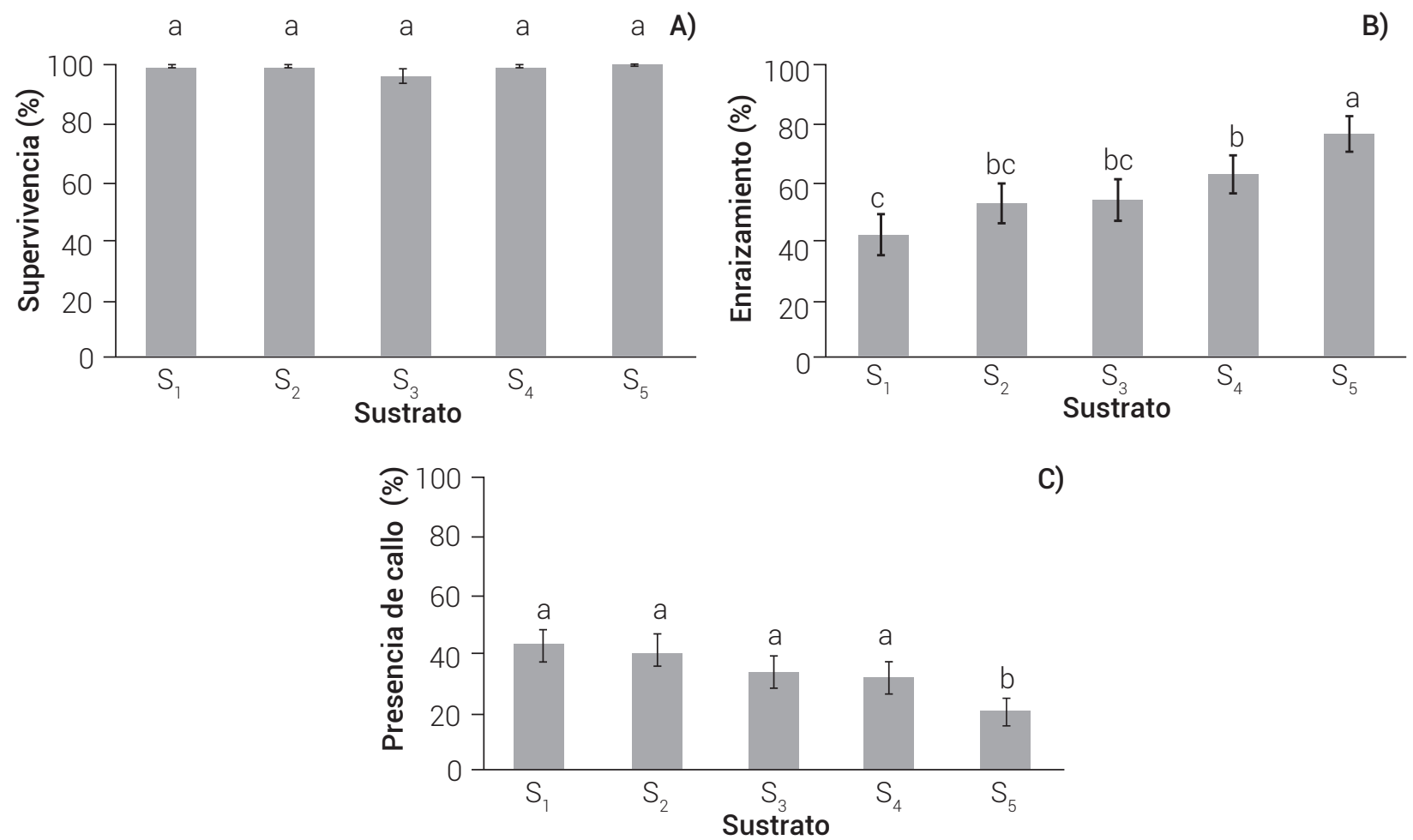

C)

Figura 1. Efecto del sustrato sobre: A) supervivencia, B) enraizamiento y C) presencia de callo. $S_{1}=1: 9, S_{2}=3: 7, S_{3}=5: 5$, $S_{4}=7: 3$ y $S_{5}=9: 1$ (proporciones aserrín y corteza v:v).

Cuadro 3. Coeficiente de correlación de las propiedades físicas y químicas del sustrato con el enraizamiento y presencia de callo en las estacas $(n=5)$.

\begin{tabular}{|c|c|c|c|c|c|c|c|c|}
\hline & Aserrín (\%) & PT (\%) & PA (\%) & $\mathrm{PRH}(\%)$ & $\begin{array}{c}\text { DA } \\
\left(\mathrm{g} \mathrm{cm}^{-3}\right)\end{array}$ & $\mathrm{pH}$ & $\begin{array}{c}C E \\
\left(d S m^{-1}\right)\end{array}$ & $\begin{array}{c}\mathrm{ClC} \\
\left(\mathrm{cmol} \mathrm{kg}^{-1}\right)\end{array}$ \\
\hline $\begin{array}{l}\text { Enraiza- } \\
\text { miento (\%) }\end{array}$ & $\begin{array}{c}0.97 * \star \\
(P=0.004)\end{array}$ & $\begin{array}{c}0.89 * \\
(P=0.040)\end{array}$ & $\begin{array}{c}-0.72 \mathrm{~ns} \\
(P=0.174)\end{array}$ & $\begin{array}{c}0.85 \mathrm{~ns} \\
(P=0.070)\end{array}$ & $\begin{array}{c}0.97 * * \\
(P=0.006)\end{array}$ & $\begin{array}{c}0.90 * \\
(P=0.034)\end{array}$ & $\begin{array}{c}-0.43 \mathrm{~ns} \\
(\mathrm{P}=0.469)\end{array}$ & $\begin{array}{c}-0.99 * * \\
(P=0.002)\end{array}$ \\
\hline Callo (\%) & $\begin{array}{c}-0.81 \mathrm{~ns} \\
(\mathrm{P}=0.100)\end{array}$ & $\begin{array}{c}-0.83 \mathrm{~ns} \\
(\mathrm{P}=0.083)\end{array}$ & $\begin{array}{c}0.92 * \\
(P=0.026)\end{array}$ & $\begin{array}{c}-0.93 * \\
(P=0.021)\end{array}$ & $\begin{array}{c}0.78 \mathrm{~ns} \\
(\mathrm{P}=0.120)\end{array}$ & $\begin{array}{c}-0.92 * \\
(P=0.026)\end{array}$ & $\begin{array}{c}-0.02 n s \\
(P=0.973)\end{array}$ & $\begin{array}{c}0.86 \mathrm{~ns} \\
(\mathrm{P}=0.065)\end{array}$ \\
\hline
\end{tabular}

PT: porosidad total, PA: porosidad de aireación, PRH: porosidad de retención de humedad, DA: densidad aparente, CE: conductividad eléctrica, CIC: capacidad de intercambio catiónico, ns: sin diferencias significativas $(P>0.05)$, * y **: diferencias significativas con $(P \leq 0.05)$ y $(P \leq 0.01)$, respectivamente.

(de 21 a $16 \%$ ). Lo anterior se explica por el hecho de que las estacas requieren de suministro de agua para evitar deshidratación, lo que depende del agua en el sustrato (Rein et al., 1991), sin llegar a condiciones anegadas (Ragonezi et al., 2010) debido a que el oxígeno es importante para el proceso de formación de la raíz (Erstad y Gislerød, 1994), de ahí que el tratamiento $\mathrm{S}_{5}$ (9:1 aserrín:corteza) con mayor humedad (70 \%) y menor porosidad de aireación (16 \%) presentó mayor enraizamiento (Cuadro 1).

Por otro lado, se observa que las propiedades físicas y químicas de los sustratos se modificaron de manera favorable al finalizar el experimento (Cuadros 1 y 2 ), principalmente en los tratamientos que presentaron menor porcentaje de enraizamiento por las razones antes mencionadas, lo que sugiere que se pueden reutilizar los sustratos con contenidos de corteza altos $\left(S_{1}\right.$ y $\left.S_{2}\right)$, ya que podrían generar condiciones adecuadas para el siguiente ciclo de enraizamiento de estacas.

\section{CONCLUSIONES}

La mezcla 9:1 de aserrín y corteza de pino cuenta con características físicas y químicas apropiadas que 
Cuadro 4. Medias ( \pm error estándar), por tipo de sustrato, de las características de las raíces generadas.

\begin{tabular}{lccc}
\hline $\begin{array}{l}\text { Sustratos } \\
\text { (aserrín:corteza) }\end{array}$ & \multicolumn{2}{c}{ Raíces primarias } & $\begin{array}{c}\text { Estacas con raíces } \\
\text { secundarias (\%) }\end{array}$ \\
\cline { 2 - 3 } $\mathrm{S}_{1}(1: 9)$ & Número & Longitud $(\mathrm{cm})$ & $69.4 \pm 6.2 \mathrm{a}$ \\
$\mathrm{S}_{2}(3: 7)$ & $1.7 \pm 0.17 \mathrm{a}$ & $5.9 \pm 0.38 \mathrm{a}$ & $71.0 \pm 6.1 \mathrm{a}$ \\
$\mathrm{S}_{3}(5: 5)$ & $1.7 \pm 0.17 \mathrm{a}$ & $5.8 \pm 0.38 \mathrm{a}$ & $72.8 \pm 6.0 \mathrm{a}$ \\
$\mathrm{S}_{4}(7: 3)$ & $2.1 \pm 0.19 \mathrm{a}$ & $5.3 \pm 0.37 \mathrm{a}$ & $62.9 \pm 6.5 \mathrm{a}$ \\
$\mathrm{S}_{5}(9: 1)$ & $1.9 \pm 0.18 \mathrm{a}$ & $5.4 \pm 0.37 \mathrm{a}$ & $76.1 \pm 5.7 \mathrm{a}$ \\
\hline
\end{tabular}

Medias con letras iguales en la misma columna no son estadísticamente diferentes (Tukey, P $\leq 0.05$ ).

permitieron un porcentaje elevado de enraizamiento de estacas de Pinus patula, mientras que la mezcla con proporción mayor de corteza de pino (90 \%) presentó los valores más bajos de enraizamiento de estacas.

\section{BIBLIOGRAFÍA}

Abad B. M., P. Noguera y B. C. Carrión (2004) Los sustratos en los cultivos sin suelo. In: Tratado de Cultivo sin Suelo. Segunda edición. M. Urrestarazu G. (coord.). Mundi-Prensa. Madrid, España. pp:113-158, https://doi.org/10.13140/RG.2.1.4853.2645

Aguilera-Rodríguez M., A. Aldrete, T. Martínez-Trinidad y V. M. Ordáz-Chaparro (2016) Producción de Pinus montezumae Lamb. con diferentes sustratos y fertilizantes de liberación controlada. Agrociencia 50:107-118

Alcantara G. B., L. L. F. Ribas, A. R. Higa, K. C. Z. Ribas e H. S. Koehler (2007) Efeito da idade da muda e da estação do ano no enraizamento de miniestacas de Pinus taeda L. Revista Árvore 31:399-404, https://doi.org/10.1590/S0100-67622007000300005

Aparicio-Rentería A., S. F. Juárez-Cerrillo y L. R. Sánchez-Velásquez (2014) Propagación por enraizamiento de estacas y conservación de árboles plus extintos de Pinus patula procedentes del norte de Veracruz, México. Madera y Bosques 20:85-96, https://doi. org/10.21829/myb.2014.201178

Bielenin M. (2003) Rooting and gas exchange of conifer cuttings treated with indolebutyric acid. Journal of Fruit and Ornamental Plant Research 11:99-105.

Browne R. D., C. G. Davidson and S. M. Enns (2000) Improvements in asexual multiplication procedures for jack pine (Pinus banksiana). New Forests 19:259-278, https://doi. org/10.1023/A:1006797705238

Erstad J. L. F. and H. R. Gislerød (1994) Water uptake of cuttings and stem pieces as affected by different anaerobic conditions in the rooting medium. Scientia Horticulturae 58:151-160, https://doi. org/10.1016/0304-4238(94)90135-X

Escobar-Sandoval M. C., J. J. Vargas-Hernández, J. López-Upton, S. EspinosaZaragoza y A. Borja-de la Rosa (2018) Parámetros genéticos de calidad de madera, crecimiento y ramificación en Pinus patula. Madera y Bosques 24:e2421595, https://doi.org/10.21829/ myb.2018.2421595

Fain G. B., C. H. Gilliam, J. L. Sibley, C. R. Boyer and A. L. Witcher (2008) Whole tree substrate and fertilizer rate in production of greenhousegrown petunia (Petunia $\times$ hybrida Vilm.) and marigold (Tagetes patula L.). HortScience 43:700-705, https://doi.org/10.21273/ hortsci.43.3.700

Farjon A., J. A. Pérez de la Rosa and B. T. Styles (1997) A Field Guide to the Pines of Mexico and Central America. Royal Botanic Gardens Kew. Oxford, England. 147 p.

Fonteno W. C. and T. E. Bilderback (1993) Impact of hydrogel on physical properties of coarse-structured horticultural substrates. Journal of the American Society for Horticultural Science 118:217-222, https://doi.org/10.21273/jashs.118.2.217

Fregoso-Madueño J. N., J. R. Goche-Télles, J. G. Rutiaga-Quiñones, R. F.
González-Laredo, M. Bocanegra-Salazar and J. A. Chávez-Simental (2017) Alternative uses of sawmill industry waste. Revista Chapingo Serie Ciencias Forestales y del Ambiente 23:243-260, https://doi.org/10.5154/r.rchscfa.2016.06.040

Goldfarb B., S. E. Surles, M. Thetford and F. A. Blazich (1998) Effects of root morphology on nursery and first-year field growth of rooted cuttings of loblolly pine. Southern Journal of Applied Forestry 22:231-234, https://doi.org/10.1093/sjaf/22.4.231

Hamann A. (1998) Adventitious root formation in cuttings of loblolly pine (Pinus taeda L.): developmental sequence and effects of maturation. Trees 12:175-180, https://doi.org/10.1007/ PL00009707

Hartmann H. T., D. E. Kester, F. T. Davies and R. L. Geneve (2014) Plant Propagation: Principles and Practices. Eighth edition. PrenticeHall. Upper Saddle River, New Jersey, USA. 922 p.

Henrique A., E. N. Campinhos, E. O. Ono and S. Z. de Pinho (2006) Effect of plant growth regulators in the rooting of Pinus cuttings. Brazilian Archives of Biology and Technology 49:189-196, https://doi.org/10.1590/S1516-89132006000300002

King A. R., M. A. Arnold, D. F. Welsh and W. T. Watson (2011) Substrates, wounding, and growth regulator concentrations alter adventitious rooting of baldcypress cuttings. HortScience 46:1387-1393, https://doi.org/10.21273/HORTSCI.46.10.1387

Majada J., C. Martínez-Alonso, I. Feito, A. Kidelman, I. Aranda and R. Alía (2011) Mini-cuttings: an effective technique for the propagation of Pinus pinaster Ait. New Forests 41:399-412, https://doi. org/10.1007/s11056-010-9232-x

Maher M., M. Prasad and M. Raviv (2008) Organic soilless media components. In: Soiless Culture: Theory and Practice. M. Raviv and J. H. Lieth (eds.). Elsevier. Amsterdam, The Netherlands. pp:459-504, https://doi.org/10.1016/B978-0444529756.50013-7

Mathers H. M., S. B. Lowe, C. Scagel, D. K. Struve and L. T. Case (2007) Abiotic factors influencing root growth of woody nursery plants in containers. HortTechnology 17:151-162, https://doi. org/10.21273/HORTTECH.17.2.151

Méndez-Neri M., C. Ramírez-Herrera, J. J. Vargas-Hernández, T. MartínezTrinidad, J. López-Upton y P. A. López (2020) Diversidad genética en dos huertos semilleros de Pinus patula Schiede ex Schltdl. et Cham. Revista Fitotecnia Mexicana 43:113-119, https://doi. org/10.35196/rfm.2020.1.113-119

Rasmussen A., T. E. Smith and M. A. Hunt (2009) Cellular stages of root formation, root system quality and survival of Pinus elliottii var. elliottii $\times$ P. caribaea var. hondurensis cuttings in different temperature environments. New Forests 38:285-294, https:// doi.org/10.1007/s11056-009-9147-6

Ragonezi C., K. Klimaszewska, M. R. Castro, M. Lima, P. de Oliveira and M. A. Zavattieri (2010) Adventitious rooting of conifers: influence of physical and chemical factors. Trees 24:975-992, https://doi. org/10.1007/s00468-010-0488-8

Rein W. H., R. D. Wright and J. R. Seiler (1991) Propagation medium moisture level influences adventitious rooting of woody stem cuttings. Journal of the American Society for Horticultural Science 116:632-636, https://doi.org/10.21273/JASHS.116.4.632

Rivera-Rodríguez M. 0., J. J. Vargas-Hernández, J. López-Upton, A. VillegasMonter y M. Jiménez-Casas (2016) Enraizamiento de estacas de 
Pinus patula. Revista Fitotecnia Mexicana 39:385-392

Romo G. D., H. Navarro G., H. M. De los Santos P., O. Hernández R. y J. López U. (2014) Crecimiento maderable y biomasa aérea en plantaciones jóvenes de Pinus patula Schiede ex Schltdl. et Cham. en Zacualpan, Veracruz. Revista Mexicana de Ciencias Forestales 5:78-91, https://doi.org/10.29298/rmcf.v5i23.343

Rowe D. B., F. A. Blazich, B. Goldfarb and F. C. Wise (2002) Nitrogen nutrition of hedged stock plants of loblolly pine. II. Influence of carbohydrate and nitrogen status on adventitious rooting of stem cuttings. New Forests 24:53-65, https://doi. org/10.1023/A:1020555013964

Salaya-Domínguez J. M., J. López-Upton y J. J. Vargas-Hernández (2012) Variación genética y ambiental en dos ensayos de progenies de Pinus patula. Agrociencia 46:519-534

SAS Institute (2011) SAS/STAT® 9.3 User's Guide. SAS Institute. Cary, North Carolina. USA. 8621 p.

StumpfE. R. T., P. R. Grolli e J. A. G. da Silva (1999) Enraizamento de estacas de Chamaecyparis lawsoniana Parl. em cinco substratos com uso de ácido indolbutírico. Ciência Rural 29:207-211, https://
doi.org/10.1590/S0103-84781999000200004

Tchoundjeu Z., M. L. Ngo Mpeck, E. Assah and A. Amougou (2004) The role of vegetative propagation in the domestication of Pausinystalia johimbe (K. Schum), a highly threatened medicinal species of West and Central Africa. Forest Ecology and Management 188:175-183, https://doi.org/10.1016/j.foreco.2003.07.010

Velázquez M. A., G. Ángeles P., T. Llanderal O., A. R. Román J. y V. Reyes H. (2004) Monografía de Pinus patula. Comisión Nacional Forestal, Colegio de Postgraduados. Zapopan, Jalisco, México. 124 p.

Wallash R. (2008) Physical characteristics of soilless media. In: Soilless Culture: Theory and Practice. M. Raviv and J. H. Lieth (eds.) Elsevier. Amsterdam, The Netherlands. pp:41-116, https://doi org/10.1016/B978-044452975-6.50005-8

Witcher A. L., E. K. Blythe, G. B. Fain and K. J. Curry (2014) Stem cutting propagation in whole pine tree substrates. HortTechnology 24:30-37, https://doi.org/10.21273/HORTTECH.24.1.30

Zobel B. and J. Talbert (1984) Applied Forest Tree Improvement. Wiley. New York, USA. 505 p. 
